\title{
Fauna de anuros en la ciudad de Salta, Argentina
}

\author{
Rebeca Acosta, Rosa Vera Mesones \& Alejandro Núñez \\ Facultad de Ciencias Naturales, Consejo de Investigación, Universidad Nacional de Salta, Avda. Bolivia 5150. (4400). \\ Salta.Argentina; racosta@unsa.edu.ar, rvera@unsa.edu.ar, racosta1966@yahoo.com.ar. \\ Recibido 03-I-2005. Corregido 01-VIII-2005. Aceptado 01-VIII-2005.
}

\begin{abstract}
Anuran fauna from Salta City, Argentina. The loss and transformation of habitat by anthropogenic activities is one of the main causes of biodiversity decrease. Amphibians are declining all over the world and one of the causes of this decline is thought to be habitat reduction. However, there are anuran populations in highly disturbed habitats, like cities. This study presents the ecological baseline needed to understand anuran communities in urban environments, for Salta City, Argentina (ca. 502000 inhabitants). The sample was stratified by environments defined by infrastructure and geographic location. The species were identified by visual encounter techniques and by auditory transect during the 2002-2003 reproductive period. Three families, seven genera and twelve species were recorded and the species richness decreased from city border to center. Similarity index values among geographic areas was higher East-West (94.7) than South-West (33.3). Bufo arenarum was the only species recorded in all environments. Hyla andina and Odontophrynus americanus had a restricted distribution and are here considered vulnerable. The border effect can explain some distribution pattern in these populations. A map of the potential distribution of recorded species for Salta is provided. Rev. Biol. Trop. 53(34): 569-575. Epub 2005 Oct 3.
\end{abstract}

Key words: Inventory, anuran, urban environments, conservation.

Uno de los problemas ambientales más relevantes a escala global es la reducción de la diversidad biológica asociada a los efectos de pérdida, fragmentación y transformación del hábitat como consecuencia de las actividades humanas (Hanken 1999, Knutson et al. 1999, 2000). Entre estas actividades, la instalación de asentamientos humanos significa la pérdida total del hábitat para muchas especies; sin embargo, para otras implica el surgimiento de nuevos ambientes adecuados para la colonización y desarrollo de sus poblaciones.

En el caso particular de los anfibios, se ha sugerido que es un grupo cuyas poblaciones se encuentran en franca declinación a nivel global debido a múltiples causas, incluyendo entre otras la pérdida de los hábitat originales en interacción con restricciones filogenéticas debido a su baja capacidad de recolonizar áreas después de extinciones locales, baja movilidad relativa y filopatría (Blaustein et al. 1994, Alford y Richards 1999, Houlahan et al. 2000, Kiesecker et al. 2001).

A pesar que los asentamientos humanos constituyen una causa de peso en la pérdida de diversidad de anfibios, se observan poblaciones de anuros en ambientes altamente antropizados como las ciudades, formando parte de su identidad biológica y cultural. Al mismo tiempo, debido a sus hábitos alimentarios, constituyen, conjuntamente con algunas especies de quirópteros, un importante control biológico de las poblaciones de insectos.

En el marco de esta problemática, el éjido de la Ciudad de Salta, desde su fundación en el año 1582 hasta la actualidad ha experimentado numerosas modificaciones. En ese sentido, cumpliendo instrucciones en el marco de la concepción geoestratégica del Virrey Toledo, el fundador de esta Ciudad, Hernando de Lerma 
ubicó el éjido en el Valle de Salta, actualmente Valle de Lerma. Este sector era atravesado por varios ríos, entre ellos, el actual río Arenales, cuyo cauce experimentó varias modificaciones por desplazamientos tanto naturales como antrópicos. Asimismo, la ciudad, al estar ubicada en el sector bajo del Valle también era atravesada por numerosos arroyos. Crónicas de 1772 indican que en los canales o "tagaretes" era habitual encontrar una variada fauna ictícola. Con el paso del tiempo y el crecimiento urbano no planificado, muchos de estos sitios fueron cerrados, canalizados y/o desviados (Caseres 1989).

En este espacio geográfico la población ha crecido ininterrumpidamente, los registros históricos indican que en 1810 se contaban 10000 habitantes, en 1914, 28000 habitantes, en 1944, 400000 habitantes y en la actualidad 502316 habitantes, concentrando aproximadamente el $50 \%$ de la población total de la provincia (INDEC 2002).

Hasta la fecha no se cuenta con información sistematizada de la presencia, distribución y parámetros ecológicos básicos de las especies de anuros presentes en la ciudad de Salta. Esta situación es reflejo de la ausencia de políticas locales respecto a la conservación de la fauna de anuros. Varios pueden ser los factores que interactúan para no considerar localmente a los anuros potencialmente en peligro; entre estos están los relativos a ciertos atavismos culturales, el desconocimiento de la potencialidad ecológica y económica de los mismos, la necesidad de generar espacios productivos inmediatos y en general la desvalorización del aporte de la fauna de anfibios a la diversidad biológica local.

Considerando la problemática ambiental, tanto la referida al crecimiento poblacional humano como a las cuestiones ambientales a nivel mundial, se realizó un inventario, información de base ecológicamente importante para posteriormente diseñar medidas conservativas de la comunidad de anuros en la Ciudad de Salta.

\section{MATERIALES Y MÉTODOS}

Teniendo en cuenta el marco de desarrollo histórico del éjido urbano, los distintos sitios de muestreo fueron agrupados siguiendo dos criterios: tipo de ambiente y ubicación geográfica.

A partir de las características de la infraestructura urbana, se establecieron tres categorías o tipos de ambientes, identificados como: (A) canales, acequias u otro tipo de canalizaciones a cielo abierto; (B) trazado de vías pavimentadas con banquinas proclives a acumular agua en época estival; (C) espacios verdes destinados a plazas o sitios de recreación.

Ubicación geográfica: a partir de planos de la ciudad se establecieron cinco categorías o zonas geográficas: Norte, Oeste, Centro, Este y Sur. En cada zona se muestrearon los tres tipos de ambientes seleccionados, tres por cada uno, respetando que la extensión fuera similar a los presentes en otras zonas.

Durante la temporada reproductiva que abarcó desde Octubre de 2002 a Marzo de 2003, se realizaron en total noventa muestreos, cubriendo los meses de registro y considerando los ambientes seleccionados (tres por zona) y las zonas geográficas estudiadas (cinco en total). Se utilizó para los muestreos Técnicas de Encuentro Visual (VES) y Técnicas de Transecta Auditiva (AST) (Heyer et al. 1994). Para ambas técnicas se diseñaron transectas en faja de hasta 100 metros por 5 metros, ancho calculado a partir de la sensibilidad de registro auditivo. En el caso de los ambientes de tipo A y $\mathrm{B}$ recorriendo un tiempo determinado de una hora, mientras que para los ambientes de tipo C utilizando el diseño de"caminata al azar", también por el espacio de una hora en cada sitio. Asimismo, para el desarrollo de AST se utilizó un grabador digital marca Cénix, mediante el cual se registró por espacio de una hora por sitio y zona las vocalizaciones de los organismos presentes. Para ambas técnicas lo que se registraba era la ocurrencia de los individuos identificando la especie, en ese sentido la técnica AST colabora en la identificación, 
fundamentalmente de las especies crípticas, por caracterización de su vocalización. Se calculó la similitud entre zonas aplicando el Indice de Sorensen (Krebs 1989).

$$
I=\frac{2 c}{a+b}
$$

c = número de especies común entre las zonas comparadas.

$\mathrm{a}, \mathrm{b}=$ riquezas en cada zona.

\section{RESULTADOS}

Se detectó la presencia de tres familias, siete géneros y doce especies (Cuadro 1). Considerando la distribución de las especies en función del tipo de ambiente antropizado, se encontró que Bufo arenarum (Hensel 1867), Physalaemus cuqui (Lobo 1993), P. biligonigerus (Cope 1861) y Leptodactylus latinasus (Jiménez de la Espada 1875), están presentes en todos; mientras que Odontophrynus americanus (Duméril \& Bribon 1841) e Hyla andina (Muller 1923), sólo se registraron en uno de ellos. (Cuadro 1, Fig. 1).

\section{CUADRO 1}

Especies de anuros registrados en la ciudad de Salta y presencia $(x)$ de las mismas en función del tipo de ambiente

\begin{tabular}{|c|c|c|c|}
\hline \multirow[b]{2}{*}{ Especie } & \multicolumn{3}{|c|}{ Tipo Ambiente } \\
\hline & A & B & $\mathrm{C}$ \\
\hline Bufo arenarum & $\mathrm{x}$ & $\mathrm{x}$ & $\mathrm{x}$ \\
\hline Bufo schneideri & $\mathrm{x}$ & $\mathrm{x}$ & \\
\hline Hyla andina & $\mathrm{x}$ & & \\
\hline Scinax nasicus & $\mathrm{x}$ & $\mathrm{x}$ & \\
\hline Scinax fuscovaria & $\mathrm{x}$ & $\mathrm{x}$ & \\
\hline Leptodactylus latinasus & $\mathrm{x}$ & $\mathrm{x}$ & $\mathrm{x}$ \\
\hline Leptodactylus fuscus & $\mathrm{x}$ & $\mathrm{x}$ & \\
\hline Leptodactylus chaquensis & $\mathrm{x}$ & $\mathrm{x}$ & \\
\hline Physalaemus cuqui & $\mathrm{x}$ & & \\
\hline Physalaemus biligonigerus & $\mathrm{x}$ & $\mathrm{x}$ & $\mathrm{x}$ \\
\hline Pelurodema borelli & $\mathrm{x}$ & $\mathrm{x}$ & $\mathrm{x}$ \\
\hline Odontophrynus americanus & $\mathrm{x}$ & $\mathrm{x}$ & \\
\hline Porcentaje de especies (\%) & 100 & 83.33 & 33.33 \\
\hline
\end{tabular}

La mayor riqueza de especies correspondió al ambiente A y la menor al C (Cuadro 1). En cuanto a la distribución de especies en función de las zonas geográficas, se encontró que Bufo arenarum y Leptodactylus latinasus, presentan la mayor amplitud en el ámbito de distribución, al tiempo que Hyla andina y Odontophrynus americanus representan las especies de menor ámbito de distribución (Cuadro 2).

La riqueza de especies en función de las zonas geográficas, es mayor en el este y oeste; y menor en la zona sur. Asimismo la zona centro presenta una riqueza de $58 \%$ semejante a la de la zona norte.

La similitud entre zonas fue mayor para el par Este-Oeste (94.7), mientras que el menor fue para el par Sur-Oeste (33.3) (Cuadro 3).

\section{DISCUSIÓN}

Actualmente existe un creciente interés por analizar los ecosistemas urbanos (Hostetler y Holling 2000, Islam et al. 2004, Rubbo y Kiesecker 2005), reconociendo que en su estudio se relacionan los aspectos biológicos y aquellos provenientes de las ciencias sociales (Mcintyre et al. 2000). En el marco de una creciente preocupación por la declinación de especies a nivel mundial, los investigadores consideran apropiado utilizar datos provenientes de diversas fuentes, entre ellas, históricas como una base para comparar con información actual de los mismos sitios y evaluar así los cambios en distribuciones de presencia/ausencia. Asimismo, este tipo de procedimiento permite, re-evaluar el estado de situación y por ende extenderla en el tiempo lo suficiente como para estimar adecuadamente el valor de los datos obtenidos (Skelly et al. 2003). Si bien este aspecto a nuestro entender resulta fundamental, hasta el momento no se dispone de información alguna respecto a la riqueza de especies de anuros en la ciudad de Salta, y menos aún un inventario de las mismas, por lo que los resultados obtenidos constituyen el primer aporte y podrían servir de referente para el monitoreo de las poblaciones asentadas en el éjido urbano. 


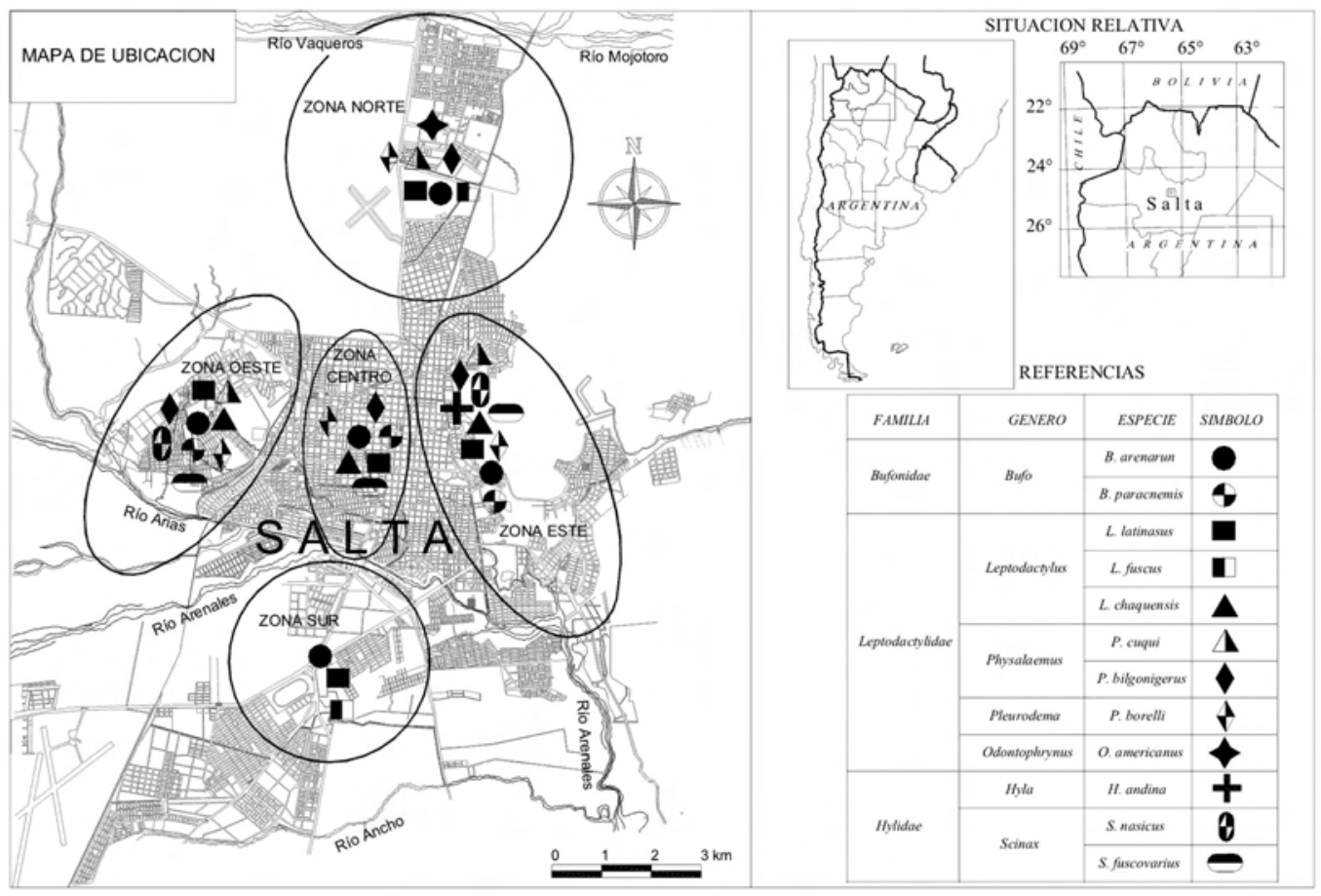

Fig. 1. Ubicación geográfica de la Ciudad de Salta, zonas estudiadas y distribución de las especies presentes. Los círculos no refieren proporciones.

CUADRO 2

Especies de anuros presentes (x) en la ciudad de Salta, por zona geográfica

ZONAS

\begin{tabular}{|c|c|c|c|c|c|}
\hline Especie & $\mathrm{N}$ & $\mathrm{C}$ & $\mathrm{E}$ & $\mathrm{O}$ & $\mathrm{S}$ \\
\hline Bufo arenarum & $\mathrm{x}$ & $\mathrm{x}$ & $\mathrm{x}$ & $x$ & $\mathrm{x}$ \\
\hline Bufo schneideri & & $\mathrm{x}$ & $\mathrm{x}$ & $\mathrm{x}$ & \\
\hline Hyla andina & & & $\mathrm{x}$ & & \\
\hline Scinax nasicus & & & $\mathrm{x}$ & $\mathrm{x}$ & \\
\hline Scinax fuscovaria & & $\mathrm{x}$ & $\mathrm{x}$ & $\mathrm{x}$ & \\
\hline Leptodactylus latinasus & $\mathrm{x}$ & $\mathrm{x}$ & $\mathrm{x}$ & $\mathrm{x}$ & $\mathrm{x}$ \\
\hline Leptodactylus fuscus & $\mathrm{x}$ & & & & $\mathrm{x}$ \\
\hline Leptodactylus chaquensis & & $\mathrm{x}$ & $\mathrm{x}$ & $\mathrm{x}$ & \\
\hline Physalaemus cuqui & $\mathrm{x}$ & & $\mathrm{x}$ & $\mathrm{x}$ & \\
\hline Physalaemus biligonigerus & $\mathrm{x}$ & $\mathrm{x}$ & $\mathrm{x}$ & $\mathrm{x}$ & \\
\hline Pelurodema borelli & $\mathrm{x}$ & $\mathrm{x}$ & $\mathrm{x}$ & $\mathrm{x}$ & \\
\hline Odontophrynus americanus & $\mathrm{x}$ & & & & \\
\hline & 58.33 & 58.33 & 83.33 & 75 & 25 \\
\hline
\end{tabular}

La conservación de la fauna de anuros resulta especialmente dificultosa debido a las características de su ciclo de vida complejo que requiere por lo tanto de un mosaico de ambientes (Pope et al. 2000). Asimismo, en los ambientes urbanos esta problemática se acentúa, debido al trazado de las carreteras, al sistema de iluminación urbana (Mazerolle 
CUADRO 3

Valores del índice de similitud de Sorensen para las zonas estudiadas en la ciudad de Salta

$\begin{array}{lccccc} & \text { NORTE } & \text { CENTRO } & \text { ESTE } & \text { OESTE } & \text { SUR } \\ \text { NORTE } & 1 & & & & \\ \text { CENTRO } & 57.1 & 1 & & & \\ \text { ESTE } & 58.8 & 82.3 & 1 & & \\ \text { OESTE } & 62.5 & 87.5 & 94.7 & 1 & \\ \text { SUR } & 60 & 40 & 30.7 & 33.3 & 1\end{array}$

2004, Longcore y Rich 2004) y a la ausencia en muchos casos de vías de conexión entre los sitios oportunos para el desarrollo larval y la posterior dispersión de los jóvenes adultos. Este aspecto combinado con lo dificultoso que resulta detectar la dispersión a larga distancia de algunos anfibios (Bradford et al. 2003) complica el análisis de las comunidades presentes en un área urbana. En ese sentido la amplitud en el ámbito de distribución como la baja especificidad detectadas tanto para Bufo arenarum como Leptodactylus latinasus podría ser explicado por una alta capacidad de movilidad relativa y el poltipismo de estas especies lo que propiciaría mayor oportunidad de colonización, esto facilitado por las vías de contacto entre zonas debido al trazado de las canalizaciones en el éjido urbano las que desaguan finalmente en el río Arenales.

Cuando se estudian los microambientes disponibles en sectores urbanizados, es necesario considerar que los mismos presentan una pérdida de complejidad por estar sometidos permanentemente a la intervención humana (Burger et al. 2004). Así, el patrón de riqueza observado podría ser explicado desde la perspectiva que los ambientes de tipo $\mathrm{A}$, es decir las canalizaciones a cielo abierto, presentan mayor continuidad tanto espacial como temporal asegurando permanencia y movilidad por vías de contacto con la región de borde lindante a ambientes naturales los cuales presentan microcuencas adecuadas para el desarrollo de poblaciones fuente. Mientras que los ambientes de tipo B son sectores temporarios y altamente fluctuantes, dependiendo estrictamente de las precipitaciones, y los de tipo $\mathrm{C}$ están sujetos a un fuerte impacto antrópico por intervención directa (remoción de agua, mantenimiento de césped, etc.).

La mayor riqueza observada en las zonas este y oeste del éjido urbano de la ciudad de Salta podría ser explicada desde la perspectiva que estos sectores representan zonas de contacto entre ambientes naturales y altamente urbanizados, en ese contexto, las zonas de borde son más ricas por su heterogeneidad (Pope et al. 200) ambiental reforzado por la presencia del río arenales como corredor biológico. En ese sentido la zona centro, sector con el mayor impacto de urbanización, presenta una particularidad debido al sesgo impuesto por la presencia del río, reforzando por lo tanto, la idea de que el mismo se comportaría como una unidad de contacto entre las zonas este y oeste, más aún, considerando el alto índice de similitud calculado.

La zona sur presentó la menor riqueza explicada, probablemente, por representar efecto de borde con sectores de una importante actividad histórica agrícola lo cual perfila un espacio menos heterogéneo y por lo tanto menos proclive a presentar una riqueza importante de especies. Asimismo, la historia de uso de la tierra destinada a actividades agrícolas, que involucra tanto perturbaciones a nivel de la composición química del ambiente como de paisaje puede influir en la estructura de la comunidad (Hicks y Pearson 2003). En ese sentido la utilización de plaguicidas destinados a la agricultura podrían modificar la dinámica de las comunidades de anuros ya sea por alteración en el tiempo de metamorfosis o como consecuencia de una baja resistencia genética ocasionada por disminución de variabilidad debido a migraciones limitadas entre poblaciones (Boone et al. 2001, Bridges 
y Semlitsch 2001, Boone et al. 2004). Sin embargo, y desde la perspectiva de un desarrollo sustentable, es oportuno mencionar que un manejo adecuado de estos sistemas podría conducir al mantenimiento de poblaciones de anuros (Knutson et al. 2004).

Es interesante destacar que el inventario de anuros en el Valle de Lerma (Vera Mesones, comunicación personal) es similar al detectado en el éjido urbano, por lo que se podría inferir, prima facie, que la ciudad no se comportaría como una barrera insalvable para estas poblaciones, ya que por las características fisiográficas de la unidad donde se ubica la ciudad, se imponen vías de contacto entre los sectores norte y sur del Valle de Lerma mediante el trazado de las canalizaciones y entre la zona este y oeste con el río Arenales como corredor biológico.

Este aspecto no resulta menor ya que los anfibios son vulnerables a la fragmentación del hábitat, tanto por las características de su ciclo de vida como por la dinámica que se establece a nivel metapoblacional, (Knutson et al. 2000) y para preservar la diversidad de anuros en ambientes urbanos es necesario considerar asimismo la diversidad regional (Rubbo y Kiesecker 2005). Así, es fundamental avanzar hacia el conocimiento de la dinámica y estructura de las comunidades establecidas en el éjido urbano con el objeto de monitorear a posteriori el estado de las mismas y generar acciones tendientes a reforzar aquellos aspectos positivos en la conservación de la fauna de anuros.

\section{AGRADECIMIENTOS}

Agradecemos a Paola Peltzer y Rafael Lajmanovich, quienes desinteresadamente realizaron valiosos aportes al manuscrito.

\section{RESUMEN}

La pérdida y transformación del hábitat generado por actividades humanas es una de las principales causas de disminución de biodiversidad. Los anfibios constituyen un grupo en franca declinación a nivel global debido a múltiples motivos, entre ellos la reducción de sus hábitats; sin embargo, se observan poblaciones de anuros en ambientes altamente antropizados como las ciudades. El objetivo del trabajo fue recabar información de base ecológicamente necesaria para comprender la distribución de las comunidades de anuros en estos ambientes. Para ello, se efectuó el inventario de la fauna de anuros del ejido urbano de la Ciudad de Salta, Argentina, con una población estimada en 502316 habitantes. Se diseñó el muestreo por ambientes, caracterizándolos en función del tipo de infraestructura urbana y su ubicación por zonas geográficas. La identificación de las especies se realizó a partir de técnicas de encuentro visual y transecta auditiva, abarcando la temporada reproductiva 2002-2003. Se detectaron tres familias, siete géneros y doce especies, observando una disminución de la riqueza desde los bordes hacia el centro del ejido urbano. El índice de similitud entre zonas geográficas fue mayor para el par Este-Oeste (94.7), mientras que el menor fue para el par Sur-Oeste (33.3). Bufo arenarum fue la especie presente en todos los ambientes caracterizados, mientras que Hyla andina y Odontophrynus americanus son las especies con distribución más restringida y por lo tanto más vulnerables. Con los datos obtenidos, se generó un mapa de distribución potencial de las especies en la ciudad analizando las posibles causas de las diferencias observadas.

Palabras clave: Inventario, anuros, ambientes urbanos, conservación.

\section{REFERENCES}

Alford, R. \& S. Richards. 1999. Global Amphibian Declines: A Problem in Applied Ecology. Annu. Rev. Ecol. Syst. 30: 133-165.

Blaustein, A.R., Wake D.B. \& W. Sousa. 1994. Amphibian Declines: Judging Stability, Persistence \& Susceptibility of Populations to Local \& Global Extinctions. Conserv. Biol. 8: 60-71.

Boone, M.D. \& R.D. Semlitsch. 2001. Interactions of an insecticide with larval density \& predation in experimental amphibian communities. Conserv. Biol. 15: 228-238.

Boone, M.D., R.D. Semlitsch, J.F. Fairchild \& B.B. Rothermel. 2004. Effects of an insecticide on amphibians in large scale experimental ponds. Ecol. Applic. 14: 685-691.

Bradford, D.F., A.C. Neale, M.S. Nash, D.W. Sada \& J.R. Jaeger. 2003. Habitat match occupancy by toads (Bufo punctatus) in a Naturally fragmented desert landscape. Ecology 84: 1012-1023.

Bridges, C.M. \& R.D. Semlitsch. 2001. Genetic variation in insecticide tolerance in a population of southern leopard frogs (Rana sphenocephala): Implications fos amphibian conservation. Copeia 1: 7-13. 
Burger, J., C. Jeitner, H. Jensen, M. Fitzgerald, S. Carlucci, S. Shukla, S. Burke, R. Ramos \& M. Gochfeld. 2004. Habitat use in basking northern water (Nerodia sipedon) \& eastern garter (Thamnophis sirtalis) snakes in urban New Jersey. Urb. Ecosyst. 7(1): 17-27.

Caseres, M.A. 1989. Río Arenales. Su origen, su importancia y su contaminación. Revista Crecer. Salta, s.p.

Hanken, J. 1999. Why are there so many new amphibian species when amphibians are declininig?. TREE 14 (1): 7-8.

Heyer, W.R., M.A. Donnelly, R.W. McDiarmid, L.A. Hayek \& M.S. Foster (eds.). 1994. Measuring \& Monitoring Biological Diversity. Standard Methods for Amphibians. Smithsonian Institution, Washington, D.C. 364 p.

Hicks, N.G. \& S.M. Pearson. 2003. Salamnder diversity \& abundance in forests with alternative land use histories in the Southern Blue Ridge Mountains. For. Ecol. Manag. 177: 177-130.

Hostetler, M. \& C.S. Holling. 2000. Detecting the scales at which respond to structure in urban landscapes. Urb. Ecosyst. 4 (1): 25-54.

Houlahan, J. E., C.S. Findlay, B.R. Schmidt, A.H. Meyer \& S.L. Kuzmin. 2000. Quantitative evidence for global amphibian population declines. Nature 404: 752-755.

Islam, S., M.T.H. Chowdhury \& M. Rahman. 2004. Urban \& peri-urban aquaculture as an immediate source of food fish: Perspectives of Dhaka City, Bangladesh. Urb. Ecosyst. 7: 341-359.

INDEC. 2002. Encuesta Permanente de Hogares. Salta, Argentina.

Kiesecker, J.M., A.R. Blaustein \& L.K. Belden. 2001. Complex causes of amphibian population declines. Nature 410: 681-684.
Knutson, M., J.R. Sauer, D.A. Olsen, M.J. Mossman, L.M. Hemesath \& M.J. Lannoo. 1999. Effects of landscape composition \& wetland fragmentation on frog \& toad abundance \& species richness in Iowa \& Wisconsin, USA. Conserv. Biol. 13 (6): 1437-1446.

Knutson, M., J.R. Sauer, D.A. Olsen, M.J. Mossman, L.M. Hemesath \& M.J. Lannoo. 2000. Landscape associations of Frog \& Toad species in Iowa \& Wisconsin, USA. J. Iowa Acad. Sci. 107(3): 134-145.

Knutson, M.G., W.B. Richardson, D.M. Reineke, B.R. Gray, J.R. Parmelee \& S.E. Weick. 2004. Agricultural ponds support amphibian populations. Ecol. Applicat. 14: $669-684$

Krebs, C.J. 1989. Ecological Methodology. Harper \& Row, Nueva York, Nueva York, EEUU.

Longcore, T. \& C. Rich. 2004. Ecological ligth pollution. Ecol. Envirom. 2: 191-198.

Mazerolle, M.J. 2004. Amphibian road mortality in response to nightly variations in traffic intensity. Herpetologica 60 (1): 45-53.

Mcintyre, N.E., Knowles-Yánez K. \& D. Hope. 2000. Urban ecology as an interdisciplinary field: differences in the use "urban" between the social and natural sciences. Urb. Ecosyst. 4 (1): 5-24.

Pope, S.E., L. Fahrig \& H.G. Merriam. 2000. Landscape complementation and metapopulation effects on leopard frogs population. Ecology 81: 2498-2508.

Rubbo, M.J. \& J.M Kiesecker. 2005. Amphibian breeding distribution in an urbanized landscape. Conserv. Biol. 19: 504-511.

Skelly, D.K., K.L. Yurewicz, E.E. Werner \& R.A. Relyea. 2003. Estimating decline and distributional change in amphibians. Conserv. Biol. 17: 744-751. 
